\title{
ESTUDIO COMPARATIVO DEL USO DE DOS TECNOLOGÍAS COMO FACTOR DE CALIDAD \\ EN EL PROCESAMIENTO DE QUINUA (CHENOPODIUM QUINOA WILLD)
}

Percy Arapa Carcasi*

Universidad Nacional de Juliaca

Recibido: 11 de mayo del 2018 / Aprobado: 4 de septiembre del 2018

doi: 10.26439/ing.ind2019.n037.4544

RESUMEN: En este artículo se muestran los resultados de la comparación del uso de tecnología de sistema no continuo y tecnología de sistema continuo en cuanto a la calidad obtenida en ambos procesos, obteniendo la tecnología de sistema no continuo, quinua de categoría 2 con un rendimiento del $86 \%$ y la tecnología de sistema continuo, quinua de categoría 1 con un rendimiento del $96 \%$; una diferencia significativa entre ambos sistemas.

Palabras clave: quinua / control de calidad / tecnología de sistema continuo / tecnología de sistema no continuo

\section{A COMPARATIVE STUDY OF THE USE OF TWO TECHNOLOGIES AS A QUALITY FACTOR IN QUINOA (CHENOPODIUM QUINOA WILLD) PROCESSING}

ABSTRACT: This article shows the results of the comparison of using a non-continuous system technology and a continuous system technology in terms of the quality obtained in both processes. The non-continuous system technology produces second class quinoa with a yield of $86 \%$, and the continuous system technology produces first class quinoa with a yield of $96 \%$, which represents a significant difference between both systems.

Keywords: quinoa / quality control / continuous system technology / non-continuous system technology

\footnotetext{
*percyarapa@gmail.com
} 


\section{INTRODUCCIÓN}

La importancia de la quinua, según Carvajal (2011), está en "que, por sus características nutricionales, nutracéuticas y organolépticas, ha pasado a ser de un alimento para marginados a un elemento de alto valor en la alimentación humana en el ámbito mundial", siendo "necesario darle la importancia en la investigación desde el punto de vista agroindustrial para realizar el uso adecuado de sus enormes potencialidades, a través de un transformación industrial que permita valorar verdaderamente este producto" (Dirección Regional de Agricultura, 2016, p. 40). En 1996, la quinua fue catalogada por la Organización de las Naciones Unidas para la Alimentación y la Agricultura (FAO) como uno de los cultivos prometedores de la humanidad no solo por sus grandes propiedades benéficas y por sus múltiples usos, sino también por considerarla como una elección para solucionar los graves problemas de nutrición humana (FAO, 2011, p. 32). "En el año 2012, la producción de quinua en Puno fue de 32 004,1 toneladas, de las cuales el $50 \%$ fue para autoconsumo, el 10 \% se derivó a la agroindustria local y regional" (Guzmán, 2013, p. 109) y cada año es mayor el porcentaje destinado para la agroindustria.

Cabe destacar que la tecnología de procesamiento de quinua ha evolucionado, desarrollándose maquinaria específica, como las lavadoras de quinua, el seleccionador de quinua y la escarificadora de quinua, y que el conjunto de criterios de calidad del producto está "asociado con el color de los granos, contenido de proteína del grano, contenido de saponina del grano, tamaño de grano y otros que demande el mercado y el uso final de la quinua" (Gómez y Aguilar, 2016, p. 27). Las características que deben reunir los granos de quinua procesada (beneficiada) en el momento de su comercialización: la calidad, clasificación, tolerancia y presentación (Indecopi, 2009), lo que requiere el mercado para la satisfacción del consumidor.

La demanda nacional e internacional de la quinua se incrementaría en los próximos años debido al reconocimiento de su calidad nutritiva, según información recogida de los productores de quinua tanto en la región Puno como en la región Arequipa (IICA, 2005). La calidad resulta agente clave para fomentar el consumo y una visión mercantilista del cultivo de quinua, es decir, ver al cultivo de la quinua como un negocio rentable para el agricultor, articulando a la quinua en la gobernanza de la cadena del valor (OlarteCalsina, Olarte-Daza y Schultz, 2016).

La Organización de Estados Iberoamericanos para la Educación, la Ciencia y la Cultura (2012) describe así a la ciencia, la tecnología y la innovación:

El conocimiento científico y tecnológico es una de las principales riquezas de las sociedades contemporáneas y un elemento indispensable para impulsar el desarrollo económico y social. La ciencia, la tecnología y la innovación se han convertido en herramientas necesarias para la transformación de las estructuras productivas, la explotación racional de los recursos naturales, el cuidado de la salud, la alimentación, la educación y otros requerimientos sociales. (p. 6) 
El binomio tecnología e innovación "se ha convertido en el nuevo paradigma económico para triunfar en el mundo actual, por lo que empresas y países hacen esfuerzos para alcanzar las capacidades y competencias que les proporcionen ventajas superiores frente a la competencia" (Peñaloza, 2007, p. 82).

Teniendo en cuenta lo anterior, el objetivo de este trabajo es la determinación de la diferencia en la calidad de quinua con el uso de tecnologías en el procesamiento, de modo que sea posible alcanzar lo establecido en los "Requisitos" de la Norma Técnica Peruana NTP 205.062 Quinua (Chenopodium quinoa Willd) (Indecopi, 2009).

\section{MATERIALES Y MÉTODOS}

El presente estudio se realizó en dos plantas de procesamiento de quinua, ambas ubicadas en el departamento de Puno, Perú. La quinua procesada es la variedad blanca de Juli; los materiales utilizados para el estudio son una balanza digital, tamices de la serie ASTM 14, 12 y 10, vernier y una lupa. Los métodos utilizados para el procesamiento de la quinua son la tecnología de sistema no continuo que se desarrolla en la empresa Servicios Agroindustriales el Altiplano S. A. C. (Arapa, 2009), produciéndose un total de $1000 \mathrm{~kg}$, y la tecnología de sistema continuo que se desarrolla en la empresa Innova Alimentos EIRL, que produjo un total de $5000 \mathrm{~kg}$ (Arapa, 2017).

\subsection{Tecnología de sistema no continuo}

El procesamiento de quinua con la tecnología de sistema no continuo (TSNC) se detalla en la figura 1. Se puede observar que se realiza por dos métodos, húmedo y seco; las operaciones unitarias son escarificado, desaponificado, secado, selección y envasado. Para el presente estudio se ha utilizado el método húmedo.

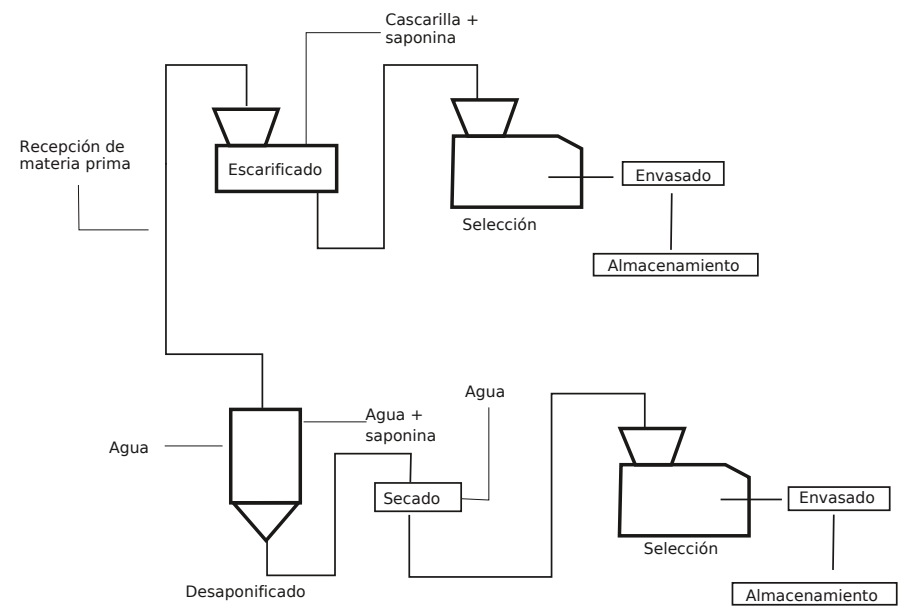

Figura 1. Proceso de producción de quinua perlada con sistema no continuo Fuente: Arapa, 2009 


\subsection{Tecnología de sistema continuo}

El procesamiento de quinua perlada con el uso de tecnología de sistema continuo (TSC) se detalla en la figura 2, en la cual se observan las operaciones unitarias de selección de impurezas, despedrado, escarificado, lavado primario, lavado secundario, lavado 1 , centrifugado, secado 1 y 2 , despedrado 1 y 2, clasificado por tamaños, selección gravimétrica, selección óptica y envasado.

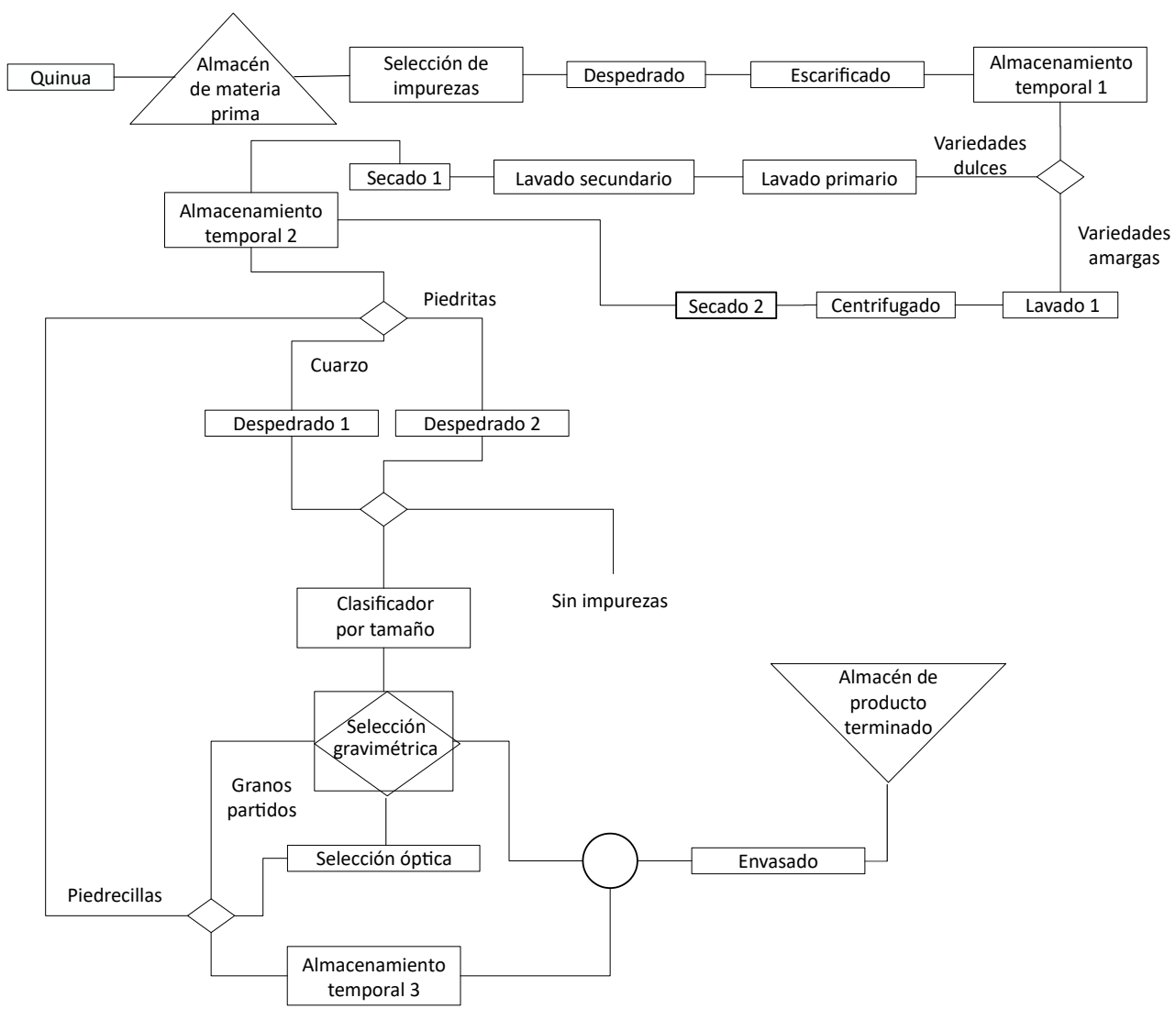

Figura 2. Proceso de producción de quinua perlada con sistema continuo

Fuente: Arapa, (2017)

\subsection{Método del estudio}

Primero se recogen las muestras de quinua perlada en la operación unitaria de envasado para ambos sistemas de producción. En segundo lugar se mide su diámetro con vernier durante distintos días de producción; se estudian las impurezas usando el tamiz; las variedades contrastantes y granos enteros se observan con lupa, y al final se pesan 
en una balanza digital. Para el estudio del rendimiento se registra el peso tomado al inicio, en el transcurso y al final de la producción. En tercer lugar se realiza el cálculo del porcentaje de variedades contrastantes utilizando la ecuación 1:

$$
\% V C=\left(\frac{\text { Gramos de } C V}{\text { Gramos de muestra }}\right) \times 100
$$

Donde \% VC es el porcentaje de variedades contrastantes y VC las variedades contrastantes.

Para el porcentaje de granos enteros se utiliza la ecuación 2:

$$
\% G E=\left(\frac{\text { Gramos de GE }}{\text { Gramos de muestra }}\right) \times 100
$$

Donde \% GE es el porcentaje de granos enteros y GE los granos enteros.

Para el porcentaje de impurezas se utiliza la ecuación 3:

$$
\% \text { Impurezas }=\left(\frac{\text { Gramos de impurezas }}{\text { Gramos de muestra }}\right) \times 100
$$

Y para el rendimiento se utiliza la ecuación 4:

$$
\text { Rendimiento }(\%)=\left(\frac{\text { Kilogramos } M P}{\text { Kilogramos } P F}\right) \times 100
$$

Donde: MP es la materia prima y PF el producto final.

En cuarto lugar se realizó el análisis estadístico con el software Minitab 18. Al final se estableció la calidad de quinua obtenida por TSC y por TSNC utilizando la NTP 205.062 (Indecopi, 2009) (tablas 1 y 2). 
Tabla 1

Determinación del tamaño de los granos de quinua en función del diámetro promedio

\begin{tabular}{lll}
\hline Tamaño de los granos & Diámetro promedio de los granos $(\mathrm{mm})$ & Malla \\
\hline Extra grandes & Mayor a 2,0 & $85 \%$ retenido en la malla ASTM 10 \\
Grandes & Mayor a 1,70 hasta 2,0 & $85 \%$ retenido en la malla ASTM 12 \\
Medianos & Mayor a 1,40 hasta 1,69 & $85 \%$ retenido en la malla ASTM 14 \\
Pequeños & Menor a 1,40 & $85 \%$ pasa por la malla ASTM 14 \\
\hline
\end{tabular}

Fuente: Indecopi, 2009

Tabla 2

Tolerancias admitidas para la clasificación de los granos de quinua en función de su grado

\begin{tabular}{|c|c|c|c|c|c|c|c|}
\hline \multirow{2}{*}{$\begin{array}{l}\text { Parámetros } \\
\text { sensoriales }\end{array}$} & \multirow[t]{2}{*}{ Unidad } & \multicolumn{2}{|c|}{ Categoría 1} & \multicolumn{2}{|c|}{ Categoría 2} & \multicolumn{2}{|c|}{ Categoría 3} \\
\hline & & Mín. & Máx. & Mín. & Máx. & Mín. & Máx. \\
\hline Granos enteros & $\%$ & 96 & & 90 & & 86 & \\
\hline Granos quebrados & $\%$ & & 1,5 & & 2,0 & & 3,0 \\
\hline Granos dañados & $\%$ & & 1,0 & & 2,5 & & 3,0 \\
\hline Granos germinados & $\%$ & & 0,15 & & 0,25 & & 0,3 \\
\hline Granos recubiertos & $\%$ & & 0,25 & & 0,30 & & 0,35 \\
\hline Granos inmaduros & $\%$ & & 0,5 & & 0,7 & & 0,9 \\
\hline Impurezas totales & $\%$ & & 0,25 & & 0,30 & & 0,35 \\
\hline $\begin{array}{l}\text { Piedrecillas en } 100 \mathrm{~g} \\
\text { de muestra }\end{array}$ & $\mathrm{U} / 100 \mathrm{~g}$ & & Ausencia & & Ausencia & & Ausencia \\
\hline Granos contrastantes & $\%$ & & 1,0 & & 2,0 & & 2,5 \\
\hline $\begin{array}{l}\text { Insectos (enteros, } \\
\text { partes o larvas) }\end{array}$ & $\%$ & & Ausencia & & Ausencia & & Ausencia \\
\hline
\end{tabular}

Fuente: Indecopi, 2009

\section{RESULTADOS Y DISCUSIONES}

En la tabla 3 se muestran los datos obtenidos en procesamiento de quinua perlada con TSC y TSNC. Las variables comparadas son las variedades contrastantes, los granos enteros, las impurezas, el diámetro y el rendimiento. 
Tabla 3

Resultados obtenidos en procesamiento de quinua perlada con el uso de sistema continuo y sistema no continuo

\begin{tabular}{ccccccccccc}
\hline N & $\begin{array}{c}\text { Variedades } \\
\text { contrastantes } \\
(\%)\end{array}$ & $\begin{array}{c}\text { Granos enteros } \\
(\%)\end{array}$ & & $\begin{array}{c}\text { Impurezas } \\
(\%)\end{array}$ & \multicolumn{2}{c}{$\begin{array}{c}\text { Diámetro } \\
(\mathrm{mm})\end{array}$} & $\begin{array}{c}\text { Rendimiento } \\
(\%)\end{array}$ \\
\cline { 2 - 10 } & TSNC & TSC & TSNC & TSC & TSNC & TSC & TSNC & TSC & TSNC & TSC \\
\cline { 2 - 10 } 1 & 1,2 & 0,9 & 90 & 97 & 0,5 & 0,2 & 1,8 & 1,7 & 86 & 96 \\
2 & 1,1 & 0,8 & 91 & 96 & 0,3 & 0,3 & 1,7 & 1,6 & 86 & 96 \\
3 & 1,4 & 0,7 & 92 & 98 & 0,2 & 0,2 & 1,9 & 1,8 & 86 & 96 \\
4 & 1,5 & 0,9 & 94 & 98 & 0,3 & 0,2 & 2 & 1,9 & 86 & 95 \\
5 & 1,2 & 0,8 & 90 & 96 & 0,2 & 0,3 & 1,7 & 1,9 & 86 & 95 \\
6 & 1,7 & 0,9 & 92 & 98 & 0,3 & 0,2 & 1,7 & 1,9 & 87 & 96 \\
7 & 1,3 & 0,6 & 93 & 97 & 0,3 & 0,2 & 1,8 & 2 & 86 & 97 \\
8 & 1,6 & 0,8 & 90 & 96 & 0,4 & 0,3 & 1,9 & 1,8 & 86 & 97 \\
9 & 1,7 & 0,7 & 92 & 97 & 0,3 & 0,2 & 1,9 & 2 & 85 & 96 \\
10 & 1,1 & 0,6 & 94 & 98 & 0,2 & 0,4 & 1,9 & 1,7 & 86 & 96 \\
\hline
\end{tabular}

Elaboración propia

En la tabla 4 se muestran los resultados de la media, la desviación típica y el error típico de la media en el procesamiento de quinua perlada con TSC y TSNC. Se observan diferencias significativas en las variables variedades contrastantes, granos enteros y rendimiento, no existiendo diferencia significativa en las variables impurezas y diámetro.

Tabla 4

Resultados de la media, la desviación típica y el error típico de la media para el procesamiento de quinua perlada con sistema continuo y sistema no continuo

\begin{tabular}{lcccccccccc}
\hline \multicolumn{1}{c}{ Variable } & $\begin{array}{c}\text { Variedades } \\
\text { contrastantes } \\
(\%)\end{array}$ & $\begin{array}{c}\text { Granos } \\
\text { enteros }(\%)\end{array}$ & & Impurezas (\%) & $\begin{array}{c}\text { Diámetro } \\
(\mathrm{mm})\end{array}$ & $\begin{array}{c}\text { Rendimiento } \\
(\%)\end{array}$ \\
\hline Tecnología & TSC & TSNC & TSC & TSNC & TSC & TSNC & TSC & TSNC & TSC & TSNC \\
Media & 0,77 & 1,38 & 97,1 & 91,8 & 0,25 & 0,3 & 1,83 & 1,83 & 96 & 86 \\
$\begin{array}{l}\text { Desviación } \\
\text { típica }\end{array}$ & 0,11 & 0,23 & 0,88 & 1,55 & 0,071 & 0,094 & 0,134 & 0,11 & 0,667 & 0,471 \\
$\begin{array}{l}\text { Error típico } \\
\text { de la media }\end{array}$ & 0,037 & 0,074 & 0,28 & 0,49 & 0,022 & 0,0298 & 0,042 & 0,33 & 0,21 & 0,15 \\
\hline
\end{tabular}

Elaboración propia 
La prueba de intervalo de confianza (IC) para dos variables y la prueba de Levene se muestran en la tabla 5, donde la relación de desviación estándar para IC es de $95 \%$, y usando Levene se muestra que en el rendimiento no se puede calcular por existir una inconsistencia en los datos obtenidos para dos variables. En las variables variedades contrastantes, granos enteros y rendimiento hay diferencias significativas en el producto obtenido con TSC en comparación con TSNC. Respecto a las variables de impurezas y diámetro del grano no hay diferencias significativas. Estos resultados se detallan en la tabla 5 , y en la figura 3 se muestra la gráfica de caja para observar con mayor claridad los resultados obtenidos de la prueba de Levene.

Tabla 5

Relación de desviación estándar y prueba de Levene para el porcentaje de variedades contrastantes, porcentaje de granos enteros, porcentaje de impurezas, diámetro de granos de quinua y rendimiento

\begin{tabular}{lcccccc}
\hline \multicolumn{1}{c}{ Variables } & \multicolumn{2}{c}{ Relación de desviación estándar } & \multicolumn{3}{c}{ Prueba de Levene } \\
\cline { 2 - 6 } & $\begin{array}{l}\text { Relación } \\
\text { estimada }\end{array}$ & $\begin{array}{c}\text { IC de 95 \% } \\
\text { para la relación } \\
\text { usando Levene }\end{array}$ & $\begin{array}{c}\text { Estadística } \\
\text { de prueba }\end{array}$ & GL1 & GL2 & Valor $\mathrm{p}$ \\
\hline \% variedades contrastantes & 2.02464 & $(1.193,4.126)$ & 7.07 & 1 & 18 & 0.016 \\
\% granos enteros & 1.7693 & $(0.773,3.568)$ & 2.32 & 1 & 18 & 0.145 \\
\% impurezas & 1.33333 & $(0.243,20.230)$ & 0.1 & 1 & 18 & 0.754 \\
Diámetro (mm) & 0.792041 & $(0.452,1.539)$ & 0.53 & 1 & 18 & 0.476 \\
Rendimiento (\%) & 0.707107 & $(*, 4.010)$ & 0.9 & 1 & 18 & 0.355 \\
\hline
\end{tabular}

Elaboración propia

Con TSC, el color de la quinua es uniforme, blanco opaco, con un diámetro de grano de quinua uniforme, sin granos partidos; con TSC y TSNC, el producto final no tiene impurezas; el manejo con TSC es más eficiente que con TSNC.

Respecto a la calidad de la quinua, Choquehuanca (1998) señala: "la presencia de granos de quinua de color negro en proporción mayor a $2 \%$ disminuye la calidad del producto" (p. 79), siendo la TSNC la que presenta mayor contenido de variedades contrastantes como la quinua de color negro, lo que afecta la calidad del producto. El diámetro del grano de quinua determinado por Calle, Del Castillo, Vargas y Bonifacio (2016) está entre 2 y 1,70 milímetros, siendo el mismo rango obtenido por ambas tecnologías. 


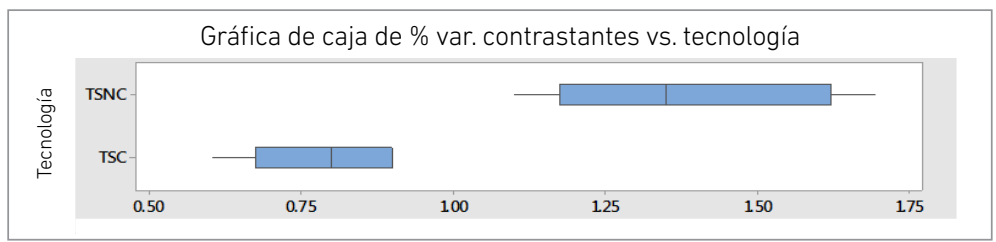

(a)

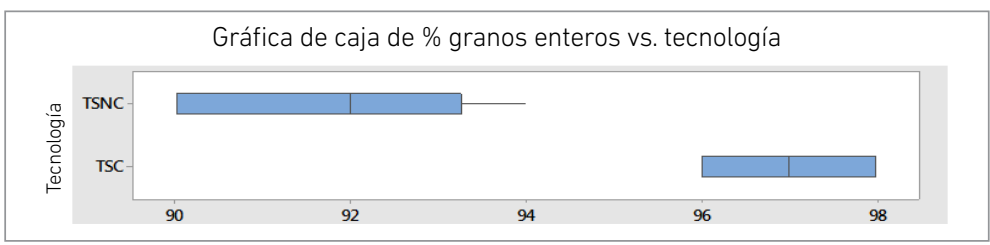

(b)

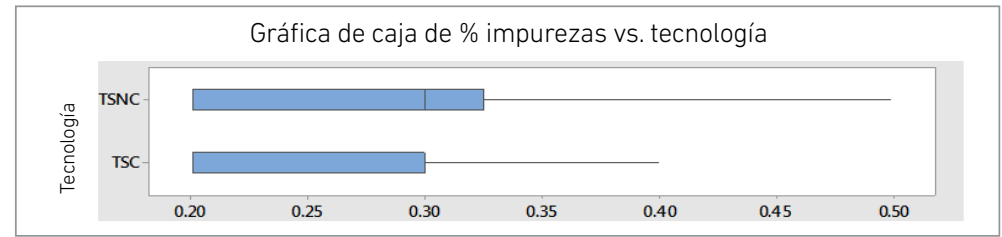

(c)

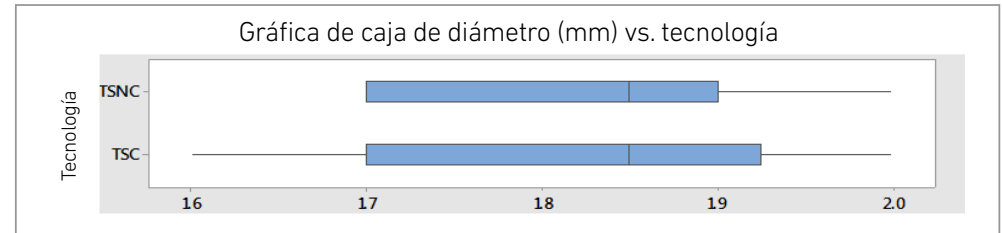

(d)

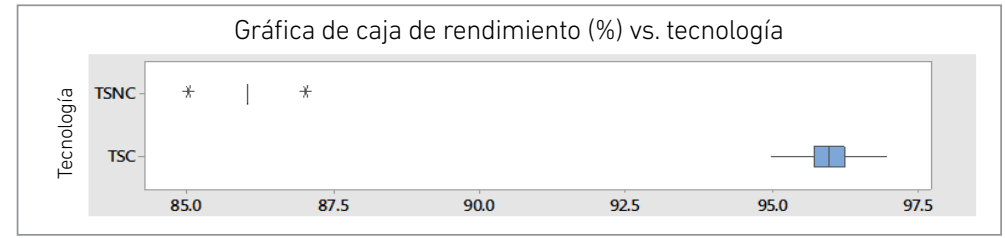

(e)

Figura 3. Gráficas de caja para el porcentaje de variedades contrastantes (a), porcentaje de granos enteros (b), porcentaje de impurezas (c), rendimiento (e) y diámetro de grano (d) Elaboración propia 


\section{CONCLUSIONES}

En el estudio se evidencia que la tecnología de sistema continuo tiene ventajas comparativas en relación con la tecnología de sistema no continuo, con una marcada diferencia en el porcentaje de variedades contrastantes, porcentaje de granos enteros y rendimiento, con una media de $86 \%$ para la TSNC y $96 \%$ para la TSC. El porcentaje de impurezas es menor en el sistema continuo, aunque no es significativa la variación. El diámetro obtenido es mayor en el sistema continuo. Confrontando los resultados con la Norma Técnica Peruana de Quinua (Chenopodium Quinoa Willd) (Indecopi, 2009), el producto obtenido con TSC está dentro de la categoría 1 y con TSNC, dentro de la categoría 2.

\section{REFERENCIAS}

Arapa, C. P. (2009). Ciencia y tecnología de la quinua. Puno: Juliaca.

Arapa, C. P. (2017). Plan HACCP quinua cañihua. Puno: Innova Alimentos EIRL.

Calle, S. L, Del Castillo, G., Vargas, M. y Bonifacio, F. A. (2016). Evaluación de características comerciales en quinua roja (Chenopodium quinoa Willd) en K'iphak'iphani, provincia Ingavi, La Paz. RIIARn 3(2), pp. 207-213.

Carvajal, S. R. (2011). La quinua y la necesidad de realizar investigación agroambiental. Tinkazos 14(30), pp. 187-190.

Choquehuanca C. F. (1998). Determinación de parámetros en la elaboración de hojuelas de quinua (Chenopodium quinoa Willd) precocida y saborizada. (Tesis de pregrado). Puno: Universidad Nacional del Altiplano.

Dirección Regional de Agricultura - Ancash. (2016). Cultivo de la quinua en Ancash. Ancash.

Gómez, P. L. y Aguilar, C. E. (2016). Guía de cultivo de la quinua. Lima: FAO/Universidad Nacional Agraria la Molina.

Guzmán, H. (2013). Competitividad de la quinua perlada para exportación: el caso de Puno. Ingeniería Industrial 31, pp. 91-112. Recuperado de https://revistas.ulima. edu.pe/index.php/Ingenieria_industrial/article/viewFile/14/14

Instituto Interamericano de Cooperación para la Agricultura (IICA) (2005). El mercado y la producción de quinua en el Perú. Lima.

Instituto Nacional de Defensa de la Competencia y de la Protección de la Propiedad Intelectual (Indecopi) (2009). Norma Técnica Peruana (NTP) 205.062 Quinua (Chenopodium quinoa Willd), Requisitos. Lima. 
Olarte-Calsina, S., Olarte-Daza C., U. y Schultz, G. (2016). La quinua en el contexto de la estandarización. Agroalimentaria 22(43), pp. 89-102.

Organización de Estados Iberoamericanos para la Educación, la Ciencia y la Cultura (2012). Ciencia, tecnología e innovación para el desarrollo y la cohesión social. Madrid.

Organización de las Naciones Unidas para la Alimentación y la Agricultura (FAO) (2011). La quinua: cultivo milenario para contribuir a la seguridad alimentaria mundial. Recuperado de http://www.fao.org/3/aq287s/aq287s.pdf

Peñaloza, M. (2007). Tecnología e innovación, factores claves para la competitividad. Actualidad Contable FACES 10(15), pp. 82-94. 
